\title{
All-Order Corrections and Multi-Jet Rates
}

\section{Jeppe R. Andersen*}

Theory Division, Physics Department, CERN, CH-1211 Geneva 23, Switzerland

E-mail: jeppe.andersen@cern.ch

\section{Jennifer M. Smillie}

Department of Physics and Astronomy, UCL, Gower Street, London, WCIE 6BT, UK

E-mail: smillie@hep.ucl.ac.uk

We discuss results from a recently proposed all-order description of hard, radiative corrections to certain multi-jet processes at hadron colliders. The description is based on obtaining an all-order estimate of the $t$-channel singularities of scattering amplitudes. As a simple example, we illustrate the similarities between $q Q$ and $q g$-scattering. In particular, we discuss how at tree-level, all nonsuppressed helicity-amplitudes for these processes consist of a pure $t$-channel pole. This structure is used in the construction of all-order approximations.

RADCOR 2009 - 9th International Symposium on Radiative Corrections (Applications of Quantum Field Theory to Phenomenology)

October 25-30 2009

Ascona, Switzerland

\footnotetext{
* Speaker.
} 


\section{Introduction}

The description of the hard perturbative corrections to any given scattering process at colliders is receiving increasing attention, necessitated by both the ever increasing number of jets entering the search channels for physics beyond the Standard Model, and the natural increasing rate at which hard pertubative corrections appear, as the energy of the collider is increased. In contrast to the situation at earlier colliders, hard pertubative corrections, giving rise to extra identifiable hard jets, will play a very significant rôle at the LHC. This is because the increase in energy allows the exploration of more of the hard multi-jet phase space, which was previously inaccessible. As an example, we illustrate in Fig. 1 the exclusive jet rates obtained in an all-order calculation of the radiative corrections to the process $p p \rightarrow h j j$ using the following minimal cuts: $k_{t}$-algorithm[1] with $\mathrm{D}=0.6, p_{t, \text { jet }}>40 \mathrm{GeV},\left|y_{j, h}\right|<4.5$. We see that the emission of each hard jet in this inclusive sample is suppressed by roughly a factor of 3-4. This ratio between the rate for a Higgs boson in

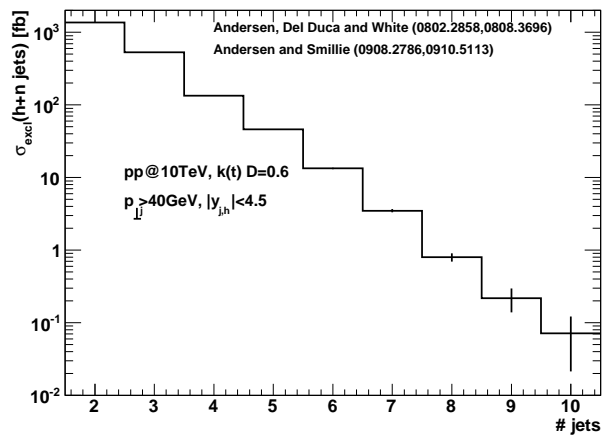

(a)

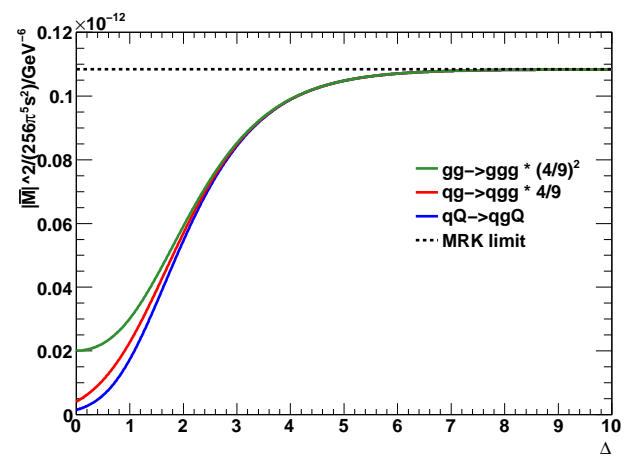

(b)

Figure 1: (a) The exclusive $n$-jet rates in $p p(10 \mathrm{TeV}) \rightarrow h j j$, as obtained in the framework of Ref.[2-5]. Monte Carlo uncertainty only. The uncertainty induced by the choice of renormalisation and factorisation scale could be evaluated directly like in Ref.[3]. (b) $|\mathscr{M}|^{2} /\left(256 \pi^{5} \hat{s}^{2}\right)$ at tree-level for $q Q \rightarrow q g Q, q g \rightarrow q g g$ and $g g \rightarrow g g g$ with the partons at $-\Delta, 0, \Delta$ in rapidity, compared with the approximation obtained from the extreme MRK limit.

association with two or three jets is consistent with estimates based on just (the inclusive) tree-level calculations of these processes. With such high rates for additional hard radiation, it is important to take these radiative corrections into account not just when estimating total rates, but especially when performing a detailed analysis of the final state, which will be needed in order to extract e.g. the $C P$-properties of the Higgs boson.

The focus is not just on radiative corrections sufficiently hard to form additional jets, but, especially when not just the total rate but the jet topology is discussed, also the (semi-) hard radiation just below the jet scale, which nevertheless changes the orientation of the hard jets. A next-toleading order calculation (the current limit whenever jets are involved) would include the first contribution to these effects. However, when studying the details of the final state for e.g. $W+\geq n$ jets, one finds a strong correlation between the jet emission and the rapidity span of the event. See e.g. Ref.[6] fig. 9 for $n=2$, where it is evident that when analysing the region of increasing rapidity span, the 3 -jet rate is much more significant than in completely inclusive analyses. This 
effect is not special to $W+$ jets, but will be seen for all processes with more than two jet, where these can exchange a gluon[7, 8, 3]. An estimate of even higher order effects is mandatory here to obtain a reliable description of the configuration of final state jets. Such an estimate can be obtained using parton shower Monte Carlos. However, this description concentrates on soft and collinear emissions, and is known to severely underestimate the effects of hard emissions. In the current contribution, we will discuss a new approach to all order corrections in processes with at least two hard jets[2-5], inspired by the behaviour of such scattering amplitudes at large invariant mass between all produced particles, the so-called Multi-Regge-Kinematic limit[9]. The formalism takes into account not just real emissions, but also the (logarithmically) leading virtual corrections in this limit, thus allowing an IR finite, all-order summation of radiative corrections.

\section{The High Energy Limit of Scattering Amplitudes from Current-Current Scattering}

The behaviour of $n$-jet production amplitudes is known in the very exclusive limit of large invariant mass between each and every jet of comparable and fixed transverse momentum (not increasing with the centre of mass energy $\hat{s}$ ). In this limit, the dominant contribution is through partonic channels, which allow for a colour octet exchange between each neighbouring (in rapidity) parton pair. In the limit, such amplitudes contribute a factor $4 g^{2} C_{A} / k_{i \perp}^{2}$ to the square of the amplitude. This leads to the following asymptotic form of the square of the tree-level scattering matrix element for the processes $g g \rightarrow g \cdots g$ :

$$
\left|\overline{\mathscr{M}}_{g g \rightarrow g \cdots g}^{M R K}\right|^{2}=\frac{4 \hat{s}^{2}}{N_{C}^{2}-1} \frac{g^{2} C_{A}}{\left|p_{1 \perp}\right|^{2}}\left(\prod_{i=2}^{n-1} \frac{4 g^{2} C_{A}}{\left|p_{i \perp}\right|^{2}}\right) \frac{g^{2} C_{A}}{\left|p_{n \perp}\right|^{2}} .
$$

The momenta $p_{1}, \ldots, p_{n}$ are here ordered according to decreasing rapidity. The same limit of $n$-jet production in $q g$-scattering ( $q Q$-scattering) is found by replacing one (two) $C_{A} \rightarrow C_{F}$. All rapidity dependence has disappeared in Eq. (2.1) (since the limit of infinite rapidity separation between all particles has been applied).

In Fig. 1(b) we compare $|M|^{2} / \hat{s}^{2}$ for $q Q \rightarrow q g Q, q g \rightarrow q g g$ and $g g \rightarrow g g g$ (the last two rescaled with appropriate powers of $C_{A} / C_{F}$ ) for a "Mercedes star" azimuthal configuration of three $40 \mathrm{GeV}$ jets, with the rapidities of the three jets chosen as $-\Delta, 0, \Delta$ respectively (see Ref.[4] for further details). It is immediately clear that 1) the square of the matrix element does tend to the right MRK limit, and 2) applying the approximate form of Eq. (2.1) in all of phase space will lead to a very poor approximation of the scattering cross section. The inability of the MRK approximation to describe the form of the full matrix element at rapidities and energies of interest to colliders is caused by some of the approximations to the kinematic invariants necessary to cast everything in terms of transverse components only.

In the following, we will describe recent progress[4, 5] in obtaining a simple approximation to the all-order $n$-jet cross section (also in association with a $W, Z$ or $h$-boson), which reproduces the full result in the MRK limit, but avoids many of the approximations which spoils the straightforward applicability of Eq. (2.1). The basic idea is to obtain a gauge invariant approximation to the $t$-channel singularities of a given scattering amplitude. We will see how that can be achieved by analysing directly the structure of amplitudes (rather than the square of these). 
Let us therefore begin by studying the simple process of $q^{-} Q^{-} \rightarrow q^{-} Q^{-}$, where $q$ and $Q$ represent different quark flavours and the negative signs represent helicity (obviously all helicity configurations lead to equally simple expressions). The only diagram is $t$-channel gluon exchange giving the following (colour and coupling stripped) matrix element:

$$
\mathscr{M}_{q^{-} Q^{-} \rightarrow q^{-} Q^{-}}=\left(\bar{u}_{1} \gamma^{\mu} u_{a}\right) \frac{g_{\mu v}}{t}\left(\bar{u}_{2} \gamma^{v} u_{b}\right) .
$$

This naturally already has an attractive form "factorised" into two currents, one which depends on the momenta of $q$ only ( $a$ and 1), and one which depends on those of $Q$ only ( $b$ and 2). This factorised form is convenient also for analysing $q g$-scattering, and generalising to $n$-parton processes (see Ref.[4] for further details). A re-arrangement of this by use of Fierz-identities would lead to spinor-products depending on the momenta of both q and Q; kinematic approximations of such spinor products would then be necessary in order to recast the (then approximated) matrix element on a factorised form (such a procedure was followed in e.g. Ref[10]), and would lead to gauge-dependence when applied to gluon scattering in the sub-MRK region.

Consider now the process $q^{-} g^{-} \rightarrow q^{-} g^{-}$, which has $s, t$ and $u$-channel contributions. However, the full, gauge-invariant scattering amplitude can be written as [5]

$$
\mathscr{M}_{q^{-} g^{-} \rightarrow q^{-} g^{-}}=e^{i \phi_{2}}\left(t_{a e}^{2} t_{e 1}^{b} \sqrt{\frac{p_{b}^{-}}{p_{2}^{-}}}-t_{a e}^{b} t_{e 1}^{2} \sqrt{\frac{p_{2}^{-}}{p_{b}^{-}}}\right) \mathscr{M}_{q^{-} Q^{-} \rightarrow q^{-} Q^{-}},
$$

where $p_{2}\left(p_{b}\right)$ is the momentum of the outgoing (incoming) gluon, which we wlog. have taken along the negative light-cone direction, $t_{i j}^{a}$ are colour matrices, and $p_{i}^{-}=p_{i_{E}}-p_{i_{z}}$ and $e^{i \phi_{2}}$ is the phase of $\left(p_{2_{x}}+i p_{2_{y}}\right)$. Again we find a fully factorised structure with just a $t$-channel pole: The difference compared with the amplitude for pure quark scattering is a factor depending on the gluon momenta only. At amplitude squared level, the result is the same as for $q Q$-scattering, but with a factor of $C_{F}$ replaced with

$$
\frac{1}{2}\left(C_{A}-\frac{1}{C_{A}}\right)\left(\frac{p_{b}^{-}}{p_{2}^{-}}+\frac{p_{2}^{-}}{p_{b}^{-}}\right)+\frac{1}{C_{A}}
$$

This momenta-dependent colour factor gives a measure of how the strength of the gluon current increases with increasing acceleration of the scattering gluon, and so we call Eq. (2.4) the Colour Acceleration Multiplier (CAM). The bigger the difference between the light-cone momenta of the incoming and outgoing gluon, the stronger the enhancement over pure quark scattering. One notes that the expression in Eq. (2.4) tends to $C_{A}$ in the limit $p_{2}^{-} \rightarrow p_{b}^{-}$(i.e. the MRK limit), and the $q g$ scattering amplitudes asymptotes to that of a rescaled $q Q$-scattering. Applications of Fierz identities would again have spoilt this easily identifiable factorisation between a quark current, and a (scaled) current depending on gluon momenta only. This is the form one finds for all of the scattering amplitudes, where the helicity of the gluon is conserved. All these amplitudes have just a $t$-channel pole. And all other helicity configurations are systematically suppressed by $\hat{s}$ in the MRK limit.

However, even for the amplitudes where the gluon helicity is flipped can the $t$-channel pole be expressed as quark currents contracted with a quantity depending on the momenta of the gluon 
only. Furthermore, the $u$ and $s$-channel pole is isolated to the case where the helicity of the incoming quark and gluon is the same, but the helicity of the outgoing gluon is flipped. Contributions unrelated to the $t$-channel pole are clearly suppressed as $\hat{s} / \hat{t} \rightarrow \infty$, and will be ignored in the following.

With the universality of the description of the $t$-channel pole established as that of a currentcurrent scattering, we can start approximating further gluon emission. This is achieved by extracting a gauge-invariant effective vertex, which takes into account soft emission from the basic $2 \rightarrow 2$ scattering process plus an emission from a connecting $t$-channel gluon. This form guarantees the right MRK limit of all the amplitudes with $n$ partons. As an example, we compare in Fig. 2 the description of the process $u g \rightarrow u g g$ at the LHC at the lowest order of the approximation and the full tree-level matrix element. Obviously, the point is not just to reproduce results, where these

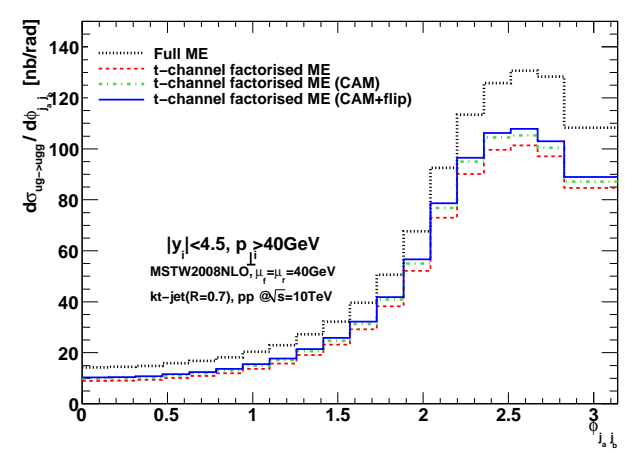

(a)

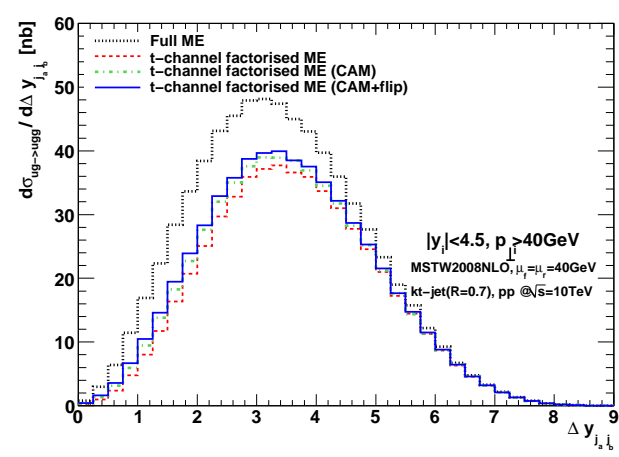

(b)

Figure 2: (a) The angular distribution between and (b) the distribution of the rapidity difference of the forward and backward jet for $q g \rightarrow q g g$ at the LHC. The coloured lines display the incremental approximations discussed in Ref.[5], with the blue line taking into account both the CAM and the $t$-channel pole from amplitudes with a flip in the gluon helicity. The solid black line is the full tree-level result taken from Madgraph [11].

can already be calculated through other means. But by illustrating how well the approximations work, where the results can be obtained through standard approaches, we hope to instill trust in the many predictions of the formalism, where these currently cannot be obtained reliably through other approaches. The quality of the approximation of hard emissions is much better than any other approach allowing for all-order estimates to be obtained. Furthermore, the all-order formulation allows for matching to full fixed order results, where these can be calculated[2].

Virtual corrections are approximated according to the Lipatov Ansatz, which has been proved to get correct even the sub-leading logarithm of the virtual corrections for the $n$-parton scattering amplitude[12]. The sum over real and virtual corrections is finite at each order in perturbation theory. And the expressions are sufficiently simple that the all-order result can be calculated as an explicit sum over the explicit integration of $n$-particle phase space. So all momenta are kept exclusive, and any analysis can be performed on these. Full details may be found in $[4,5]$, where we also discuss the description of $W, Z, h+$ jets. 


\section{Summary}

We have discussed the very basic observation of the exact factorisation of the $t$-channel pole in some $2 \rightarrow 2$ QCD processes. This forms the foundation of the scheme introduced in Ref.[4, 5], which allows for the construction of an all-order sum of parton emissions from simple $2 \rightarrow 2$ processes constructed as a sum over explicit phase space integrations over $n$-particle phase space. The sum tends to the exact all-order scattering amplitude in the limit of large invariant mass between all particles. This is the limit where the emission leads to additional jet formation. A reliable description of hard emission is not only desirable, but often critical for LHC analyses. The allorder summation of hard parton emissions allow for the direct study of e.g. jet veto efficiencies in gluon fusion $h j j$ (see Ref.[3]), where the inclusion of several, hard emissions is crucial in reaching a stable prediction. Recently, the analytic understanding of the all-order amplitude was used in designing cuts and observables[13], which will significantly aid in the extraction of the $C P$-properties of any Higgs boson created through gluon fusion.

\section{References}

[1] M. Cacciari and G. P. Salam, Dispelling the $N^{3}$ myth for the $k(t)$ jet-finder, Phys. Lett. $\mathbf{B 6 4 1}$ (2006) 57-61, [hep-ph/0512210].

[2] J. R. Andersen and C. D. White, A New Framework for Multijet Predictions and its application to Higgs Boson production at the LHC, Phys. Rev. D78 (2008) 051501, [0802 . 2858].

[3] J. R. Andersen, V. Del Duca, and C. D. White, Higgs Boson Production in Association with Multiple Hard Jets, JHEP 02 (2009) 015, [0808 . 3696].

[4] J. R. Andersen and J. M. Smillie, Constructing All-Order Corrections to Multi-Jet Rates, JHEP 01 (2010) 039, [0908.2786].

[5] J. R. Andersen and J. M. Smillie, The Factorisation of the t-channel Pole in Quark-Gluon Scattering, 0910.5113.

[6] C. F. Berger et al., Next-to-Leading Order Jet Physics with BlackHat, 0912 . 4927.

[7] J. R. Andersen, V. Del Duca, F. Maltoni, and W. J. Stirling, W boson production with associated jets at large rapidities, JHEP 05 (2001) 048, [hep-ph / 0105146 ].

[8] J. R. Andersen and W. J. Stirling, Energy consumption and jet multiplicity from the leading log BFKL evolution, JHEP 02 (2003) 018, [hep-ph / 0301081 ].

[9] L. N. Lipatov, The parton model and perturbation theory, Sov. J. Nucl. Phys. 20 (1975) 94-102.

[10] V. Del Duca, A. Frizzo, and F. Maltoni, Factorization of tree QCD amplitudes in the high-energy limit and in the collinear limit, Nucl. Phys. B568 (2000) 211-262, [hep-ph / 9909464$].$

[11] J. Alwall et al., MadGraph/MadEvent v4: The new web generation, JHEP 09 (2007) 028, [0706.2334].

[12] A. V. Bogdan and V. S. Fadin, A proof of the reggeized form of amplitudes with quark exchanges, Nucl. Phys. B740 (2006) 36-57, [hep-ph / 0601117$].$

[13] J. R. Andersen, K. Arnold, and D. Zeppenfeld, Azimuthal Angle Correlations for Higgs Boson plus Multi- Jet Events, 1001.3822. 